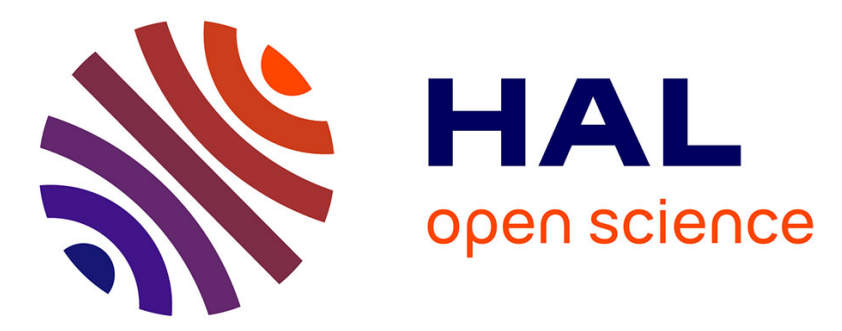

\title{
Efficient Generation of Ground Impact Probability Maps by Neural Networks for Risk Analysis of UAV Missions
}

Baptiste Levasseur, Sylvain Bertrand, Nicolas Raballand

\section{To cite this version:}

Baptiste Levasseur, Sylvain Bertrand, Nicolas Raballand. Efficient Generation of Ground Impact Probability Maps by Neural Networks for Risk Analysis of UAV Missions. 2020 International Conference on Unmanned Aircraft Systems (ICUAS), Sep 2020, Athens, Greece. pp.1398-1406, 10.1109/ICUAS48674.2020.9213935 . hal-03080144

HAL Id: hal-03080144

https://hal.science/hal-03080144

Submitted on 17 Dec 2020

HAL is a multi-disciplinary open access archive for the deposit and dissemination of scientific research documents, whether they are published or not. The documents may come from teaching and research institutions in France or abroad, or from public or private research centers.
L'archive ouverte pluridisciplinaire HAL, est destinée au dépôt et à la diffusion de documents scientifiques de niveau recherche, publiés ou non, émanant des établissements d'enseignement et de recherche français ou étrangers, des laboratoires publics ou privés. 


\title{
Efficient Generation of Ground Impact Probability Maps by Neural Networks for Risk Analysis of UAV Missions
}

\author{
B. Levasseur ${ }^{1}$, S. Bertrand ${ }^{1}$, N. Raballand ${ }^{1}$
}

\begin{abstract}
This paper investigates the generation of ground impact probability maps for UAVs in case of failure during the flight. Such maps are of a huge interest for risk assessment of UAV operations and can be used both for offline mission preparation or analysis and online decision making. Two approaches are proposed in this paper to generate such maps, taking into account a dynamical model a fixed-wing UAV and wind conditions. The first one relies on the generation of a complete database by Monte Carlo simulations. The second one is based on neural network surrogate models obtained by supervised learning using this database. Computation time required by the second approach is very small and compatible with online use. The two approaches are presented and discussed, and examples of ground impact probability maps generated are provided.
\end{abstract}

\section{INTRODUCTION}

Use of Unmanned Aerial Vehicles (UAVs) for outdoor inspection or monitoring missions has been the topic of particular attention in recent years by operators and regulatory authorities. Assessing the feasibility of such operations strongly relies on risk analysis in order to know and control the safety level in relation to infrastructures [1], critical structures, people at ground [2] [3] or road networks [4]. Mandatory to obtain flight authorizations from legal entities, risk analysis can also help in the design and preparation of the missions in order to fulfill safety constraints while optimizing their operational performances.

Although qualitative methods are still often used, such as Failure Modes and Effects Analysis (FMEA) or Functional Hazard Analyzis (FHA) [5], quantitative approaches should be implemented in order to capture the dependencies of the risks with the characteristics of the mission (characteristics of the UAV, trajectory) and its environment (population areas crossed, road networks, buildings). Such methods, including Probalistic Risk Assessment (PRA) analysis, are at the heart of the work presented in this paper.

The calculation of the risk at any instant involves a whole chain of factors intrinsic to the mission (probability of UAV failure, exposure time, ground impact probability) or external (population density, weather conditions). Determination of the probability of ground impact plays a central role in the risk analysis pipeline. It has been addressed in literature by mainly considering a particular failure mode [2]. Although many ones can occur, the work presented here focuses on main engine failure leading to an uncontrolled descent to the ground, this failure mode being actually considered as

\footnotetext{
${ }^{1}$ All authors are with Université Paris-Saclay, ONERA, Information Processing and Systems, 91123, Palaiseau, France. \{baptiste.levasseur, sylvain.bertrand, nicolas.raballand\}@onera.fr
}

one of the most probable for fixed-wing UAVs.

Several studies such as [6][7][8] have focused on the evaluation of models of descent due to engine failure, leading to analytical expressions of the impact position from a deterministic point of view for gliding or ballistic descent trajectories. However, in these works, only kinematic or reduced-dynamic models of UAVs are considered. This assumption neglects the contribution from a dynamic point of view of external factors having a strong influence on lift, such as wind, for example.

In previous work done by the authors [9] a model of uncontrolled descent to ground has been developed by considering a 6 degrees of freedom (6DoF) dynamic model of a fixedwing UAV including the effect of wind in flight mechanics. From this precise model, a first work was to consider the footprints corresponding to the extreme zones accessible by the UAV in case of glide descent. This study made it possible to identify the influence of external parameters on risk areas. Another part of this work was to implement a stochastic approach based on Monte Carlo (MC) simulation to generate descent trajectories. The impact positions obtained by this second method allowed the estimation of ground impact probability maps, taking into account several external parameters in a parametric way (wind direction and speed, aircraft altitude and speed).

For Long Range Operations (LRO), these external parameters can vary widely over the trajectory of the UAV. Since the generation of a probability map by Monte Carlo methods is a very time-consuming process, another approach has to be found to be able to compute efficiently ground impact probability maps along a whole flight trajectory. This is the contribution proposed by this paper.

A first step proposed in this paper consists in generating a consistent database of reference maps based on a relevant sampling of the space defined by these external parameters. This approach can allow offline risk assessment, e.g. for mission preparation.

One of the major drawbacks of the database is that it requires large storage and computational resources. This criterion is discriminating if one wants to be able to perform online risk assessment and to embed a risk analysis tool directly on board of the UAV. This method is also limited by the prior sampling of the available impact probability maps defined. It therefore requires interpolation methods to generate the intermediate maps if not present in the database.

To mitigate these problems, a surrogate model based on neural networks has been developed and is also presented in this paper. Once set up, this model with low computation 
burden allows to consider an online use of a risk assessment tool. This use can open up numerous applications such as real-time calculation of new mission plans according to external conditions encountered during the flight, decision making for activation of a flight termination system or for choosing an emergency landing site, etc.

This paper is organized as follows. In the following section, the methodology of stochastic impact trajectory simulations and impact probability map estimation are recalled. In Section III, the choice of parameterization and generation of a database taking into account external parameters is studied. Substitution models based on neural networks allowing online generation of impact probability maps and their interpolation are presented in Section IV. Conclusions are given in the last section of the paper.

\section{PROBABILISTIC MAP GENERATION METHODOLOGY}

\section{A. Stochastic generation of impact points}

To fully understand the parametric considerations of the ground impact probability maps and the dynamic assumptions made on the descent model, it is worth briefly recalling the map generation pipeline previously developed by the authors in [9]. The failure case considered is a total loss of control due to a power failure. In this case, at the time of the failure $t_{0}$, it is assumed that the UAV actuators are locked in equilibrium position and the thrust becomes zero. The resulting trajectory will then inevitably lead to ground impact at time $t_{f}$.

To model this behaviour, a complete 6DoF dynamic model was developed, including full flight mechanics and hence the possibility to take into account environmental parameters such as wind. The control input vector $u=$ $\left[\begin{array}{llll}\delta_{a} & \delta_{e} & \delta_{r} & \delta_{T}\end{array}\right]^{\top}$ is composed of ailerons, elevators, rudder deflections, and thrust command. The state of the dynamical system to be simulated is defined as $\chi=$ $\left[\begin{array}{llll}X^{\top} & V^{\top} & \eta^{\top} & \Omega^{\top}\end{array}\right]^{\top}$ where $X$ is the position vector defined in a local NED frame, $V$ and $\Omega$ are the translation and angular velocity vectors in the aircraft body-frame, and $\eta$ is the vector of Euler angles (roll-pitch-yaw) describing the attitude of the UAV. The wind velocity vector is denoted $V_{w}$ and can be represented in the horizontal plane by two parameters $V_{w}, \theta_{w}$ which correspond respectively to the amplitude (speed) of the wind and its direction.

The dynamic model of the UAV can be summarized with the following state-space representation.

$$
\dot{\chi}=F\left(\chi, u, V_{w}\right)
$$

The ground descent simulation is obtained by selecting an initial state vector $\chi_{0}$ as well as the control vector associated with zero thrust, $u_{0}=\left[\begin{array}{llll}\delta_{e 0} & \delta_{a 0} & \delta_{r 0} & \delta_{T 0}=0\end{array}\right]^{T}$ at a time $t_{0}$. The latter command vector will remain constant during the whole simulation. The state equation (1) is then numerically integrated until reaching an altitude $h\left(t_{f}\right)=0$ which corresponds to the point of impact on the ground. The initial condition is therefore composed of 16 parameters (state and control) that can take on a wide range of values depending on flight conditions of the UAV. However, during a steady flight (coordinated turn, cruise flight, etc.), the accessible space through these parameters is considerably reduced. In order to identify realistic initial state vectors, these should be represented in a more intuitive reduced space. This method is based on the consideration of dynamic equilibrium according to certain kinematic constraints. In this case, the control vectors and the dynamic part of the state vector are entirely defined by these constraints. A simple way to represent a trajectory is to consider two additional parameters:

- the turn rate $R=d \psi / d t$, where $\psi$ is the heading angle

- the flight path angle $\gamma=\dot{z} / V_{a}$, where $V_{a}$ is the aerodynamic speed of the aircraft and $z$ the vertical component of its position vector. Note this definition of glide angle relies on small angle assumption, motivated by the fact that the starting point is a stable glide.

Given a reduced parametrization of a steady flight, a trim algorithm [10] is then applied to retrieve the associated state parameters. This algorithm consists in cancelling the dynamic part of the equation (1) by considering the input kinematic constraints, namely the parameters $\left(V_{a}, h_{0}, R_{0}, \gamma_{0}\right)$. From these parameters, we then obtain the associated state vector $\chi_{0}$ and control vector $u_{0}$.

Real flight trajectories of an UAV performing cruise-like flight mode in straight line and constant altitude with respect to the ground were used to estimate the dispersion of the parameters $R_{0}, \gamma_{0}$ (see [9]). The distribution of this data is approximated by a Gaussian distribution and is used to sample the values $R_{0}, \gamma_{0}$. Finally, a Gaussian noise $\left(\Delta \delta_{e_{0}}, \Delta \delta_{a_{0}} \Delta \delta_{r_{0}}\right)$ is added to the equilibrium position of the actuators to model some flapping behavior during the flight (before failure instant). Let us recall that position of the actuators are then kept constant during the simulation of the descent trajectory to the ground. Wind conditions (horizontal speed and direction) are also kept constant. The simulation pipeline is represented on Figure 1.

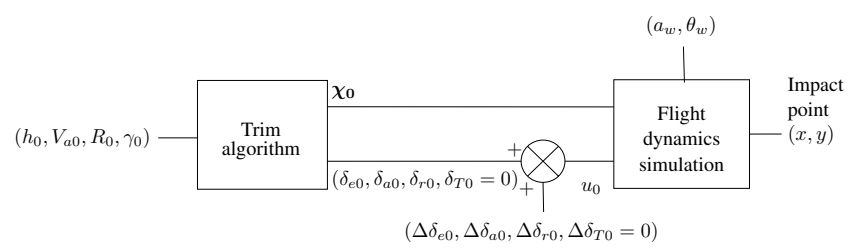

Fig. 1. Simulation flowchart used to generate ground impact points

\section{B. Probability of impact estimation}

The previously presented simulation chain involves stochastic parameters $\left(R_{0}, \gamma_{0}, \Delta \delta_{e 0}, \Delta \delta_{a 0}, \Delta \delta_{r 0}\right)$ whose distributions were identified by a priori assumptions or real data. Other parameters only depend on the environmental conditions of the UAV (wind) or correspond to a given kinematic configuration (altitude, speed). These parameters $\left(h_{0}, V_{a_{0}}, V_{w}, \theta_{w}\right)$ will be referred to later as external parameters. In order to generate a ground impact probability 
map, it is necessary to set these external parameters to given values and to generate Monte Carlo type samples of the stochastic parameters. The trajectories are then simulated until the ground collision thus generating the dataset of ground impact points. Given the complexity of the ground impact distribution and the number of stochastic parameters, 10,000 Monte Carlo runs (and thus trajectories) are generated to estimate an impact probability map. This arbitrary choice of sampling was guided by several trials.

Several methods for estimating a multivariate probability density $f$ associated with a given sample of points exist. Among these methods, there are two main categories, which are on the one hand parametric methods and on the other hand non-parametric methods [11]. Since there is no a priori knowledge on the probability distributions to be expected, non-parametric estimation is chosen.

If the histogram is at first sight the simplest solution of the non-parametric methods and has been firstly used in preliminary work [9], it lets emerge several difficulties as for the choice of the number of bins, their size and their location. Kernel density estimation proposes an alternative to overcome these problems. It also offers better theoretical results in terms of convergence [12].

The kernel density estimate is defined by

$$
\hat{f}(x, H)=n^{-1} \sum_{i=1}^{n} K_{H}\left(x-x_{i}\right)
$$

Where $\left\{x_{1}, \ldots, x_{n}\right\}$ denotes the $n$ observations (impact locations in our case), $H$ is the bandwidth matrix symmetric and positive-definite and $K_{H}(x)=|H|^{-1 / 2} K\left(H^{-1 / 2} x\right)$ and $K(\cdot)$ is a unit Gaussian kernel.

While the choice of kernel $K(\cdot)$ is not crucial, the choice of the bandwidth $H$ is very important for the performance of $\hat{f}$. This latter must be selected by optimizing an objective criterion such as mean square integrated error (MISE).

$$
\operatorname{MISE}(H)=\mathbb{E} \int_{\mathbb{R}}[\hat{f}(x, H)-f(x)]^{2} d x
$$

However in practice, it is not possible to calculate this criterion directly since it depends on the function to be estimated $f$ which is unknown. An alternative to this problem is to minimize the asymptotic approximation of MISE (AMISE) [13]:

$$
\begin{array}{r}
\operatorname{AMiSE}(H)=\frac{1}{n(4 \pi)^{d / 2}|H|^{1 / 2}} \\
+\frac{1}{4}\left(\operatorname{vech}^{\top} H\right) \Psi_{4}(\operatorname{vech} H)
\end{array}
$$

In this equation, the elements of the matrix $\Psi_{4}$ are integrated density derivative functionals, they therefore depend on the density function $f$ and its derivatives and must be estimated. The operator vech corresponds to the flattening of the triangular part of the matrix $\mathrm{H}$ (this one being symmetrical) and $d$ is the dimension of the observations. The well-known plugin-in selector [12] consists in setting up a second level of estimation by kernel with stronger assumptions using a pilot bandwidth matrix to obtain the estimate $\hat{\Psi}_{4}$. Once this quantity is estimated, it is possible to compute the AMISE criterion by replacing $\Psi_{4}$ by $\hat{\Psi}_{4}$ in the equation (4). The optimal bandwidth using the plug-in selector $H_{\mathrm{PI}}$ is finally obtained by minimizing this criterion. It is therefore this selector that was chosen for this work. The ks module in $\mathrm{R}$ [14] proposes an implementation of the calculation of the plug-in estimator and has therefore been used in this study.

\section{GENERATION OF THE DATABASE}

During LRO, it is necessary to compute the risk over distances reaching 50 to $100 \mathrm{~km}$ during which the UAV passes in many configurations. At any moment, the UAV's configuration can be identified by means of the external parameters presented in the previous section. For a given value of these parameters, it is necessary to generate 10,000 descent trajectories, the resulting ground impact points, and estimate their probability density. it is therefore impossible to envisage directly an estimation of the impact probability map at each instant.

A compromise must then be made on one hand to obtain a sufficiently accurate approximation of the ground impact probability map at a given time $t$, and on the other hand to be able to generate this map efficiently with a low computation time and memory space. The solution proposed here is based on a judicious definition and sampling of the external parameter space called $\mathcal{A}$.

It should be remembered that in the case of the steady flight presented in the map generation methodology part, there are four parameters : the initial altitude $h_{0}$, the initial speed of the UAV $V_{a_{0}}$ and the wind (parameterized by its speed $V_{w}$ and its direction $\theta_{w}$ ), by assuming the latter constant and horizontal throughout the crash trajectory. The initial position of the UAV $\left(x_{0}, y_{0}\right)$ in the horizontal plane can be ignored because results are computed in a local reference frame related to the UAV position at time of failure. Finally, the angle $\theta_{w}$, defining the wind direction with respect to the initial UAV speed vector can be limited to $[0, \pi]$ since the opposite can be found by simple symmetry.

Once set $\mathcal{A}$ is reduced to the minimum combination of variables, the database design consists in sampling it according to a regular grid. In this way, a symmetrical selection by nearest neighbours gives access to the map of interest. The limit values and sampling step sizes for each external parameter are given by the following experimental design:

- $h_{0} \in[5,150] \mathrm{m}$ with $5 \mathrm{~m}$ step size

- $V_{a_{0}} \in[20,30] \mathrm{m} / \mathrm{s}$ with $5 \mathrm{~m} / \mathrm{s}$ step size

- $V_{w} \in[0,10] \mathrm{m} / \mathrm{s}$ with $2.5 \mathrm{~m} / \mathrm{s}$ step size

- $\theta_{w} \in[0, \pi] \mathrm{rad}$ with $\pi / 5 \mathrm{rad}$ step size

The discrete subset of external parameters described above is denoted by $\mathcal{A}_{s} \subset \mathcal{A}$.

A trick is to reduce the number of simulations based on the assumption of a constant atmosphere density (no variation with respect to altitude), which is justified by the low altitudes considered. In this case, instead of generating the trajectories for each value of the initial altitude $h_{0}$ 
in the experimental design, it is thus possible to generate only a single set of trajectories starting from the altitude $h_{\max }=150 \mathrm{~m}$ which is considered by the French regulatory authorities as the maximum altitude at which a pilot can operate an UAV.

Ground impact points that would correspond to a given initial altitude $h_{0}$ are then computed as intersection points of these trajectories and the plane located at the altitude $h_{\max }-h_{0}$. Figure 2 shows an illustrative example: the grids correspond to $(x, y)$ planes at constant altitude $z$, the red curve is the UAV's path to the ground and the circles show the points of impact corresponding to a fictitious initial altitude $h_{0}=h_{\max }-z$.

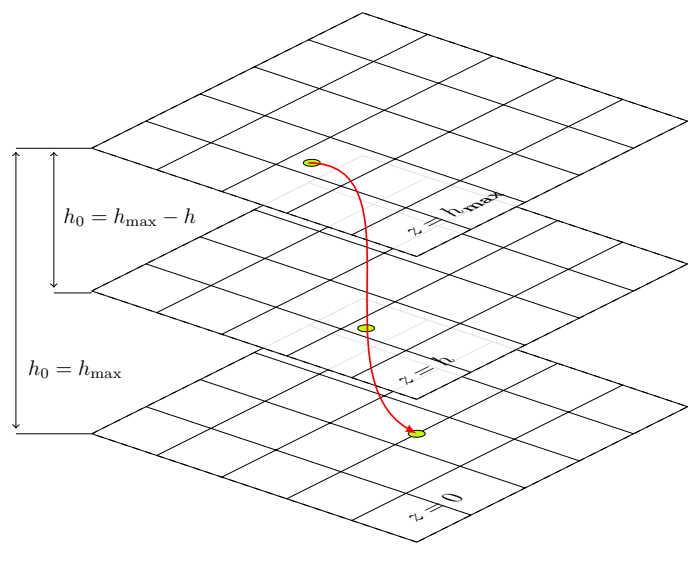

Fig. 2. Trajectory slices

Finally, only 75 sets of 10,000 simulations are required to be able to generate card $\left(\mathcal{A}_{s}\right)=2,250$ impact probability maps according to the given experiment design.

As the calculation time required to generate an impact trajectory is on the order of one second, it becomes necessary to parallelise the calculations in order to build the impact trajectory database in a reasonable amount of time. The simulations were therefore parallelized and developed on dedicated multi-core intensive computing resources. The generation of 10,000 impact trajectories for a set of external parameters was then reduced to around twenty minutes.

The final database contains all the impact points for each of the elements of $\mathcal{A}_{s}$. For each external parameters $\Theta \in \mathcal{A}_{s}$, the generation of the associated ground impact probability map consists in sampling the estimated probability density according to the desired geometric coordinates $(x, y)$.

Four examples of ground impact probability maps generated in this data set are presented to identify the influence of each of the external parameters. Throughout the rest of the paper, the initial position of the UAV will be represented by a red dot at the coordinates $(0,0)$ in the $(x, y)$ plane. Its initial speed will be directed along the $x$ axis towards the $x>0$.

On Figure $3 \mathrm{a}$ which corresponds to the no-wind case at low speed and maximum altitude, we can notice that two separate lateral modes appear in the ground impact probability map (located at $x=100 \mathrm{~m}$ and $y= \pm 100 \mathrm{~m}$ ). It is interesting to note that in this case, the probability of ground impact is

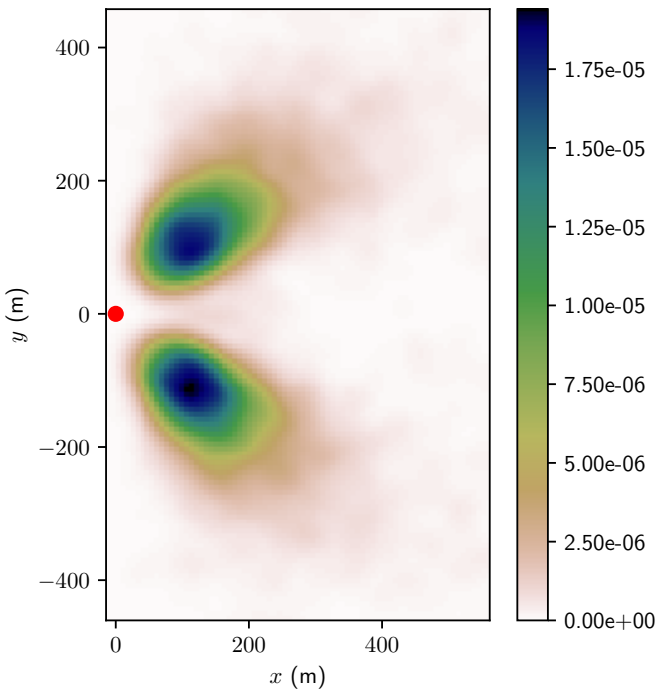

(a)

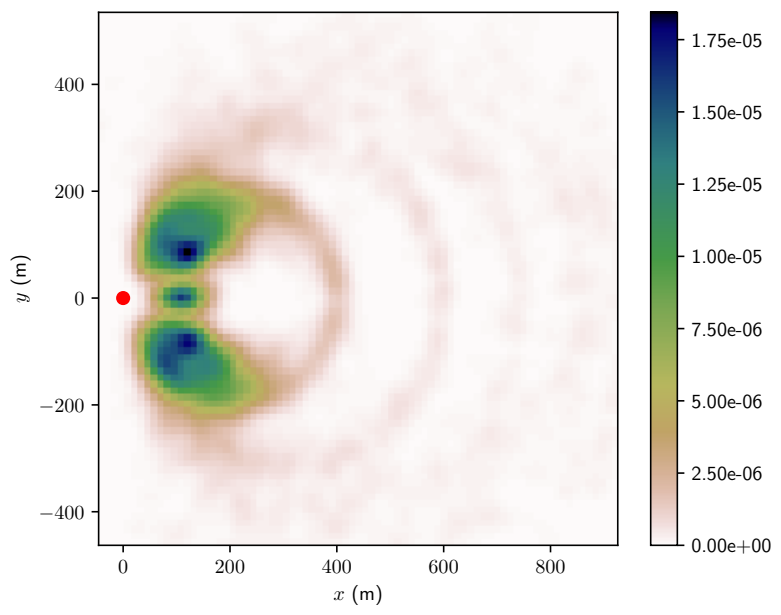

(b)

Fig. 3. Impact probability maps obtained by $\mathrm{MC}$ simulation with no wind and : (a) $h_{0}=150 \mathrm{~m}$ and $V_{a_{0}}=20 \mathrm{~m} / \mathrm{s}$, (b) $h_{0}=50 \mathrm{~m}$ and $V_{a_{0}}=20 \mathrm{~m} / \mathrm{s}$

almost zero in the area directly in front of the UAV. This distribution can be explained by the fact that trajectories to impact take a long time due to the high altitude and low speeds. The UAV therefore has time to deviate from its nominal straight glide path and begin an helical descent either to the left or to the right.

By lowering the initial position of the UAV to $50 \mathrm{~m}$, one obtains the distribution shown in Figure 3b. In this case, the two lateral modes remain present but their probability decrease, giving way to a zone of significant central impact at $x=100 \mathrm{~m}$. This modification of the impact pattern can be explained by considering that the trajectories continue a straight glide path to the ground. The higher the altitude is, the more unstable this glide path will become and will feed 


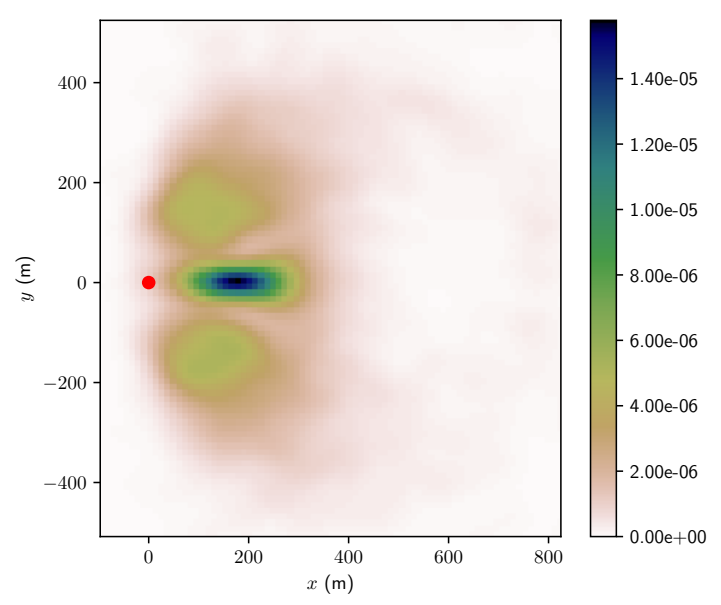

(a)

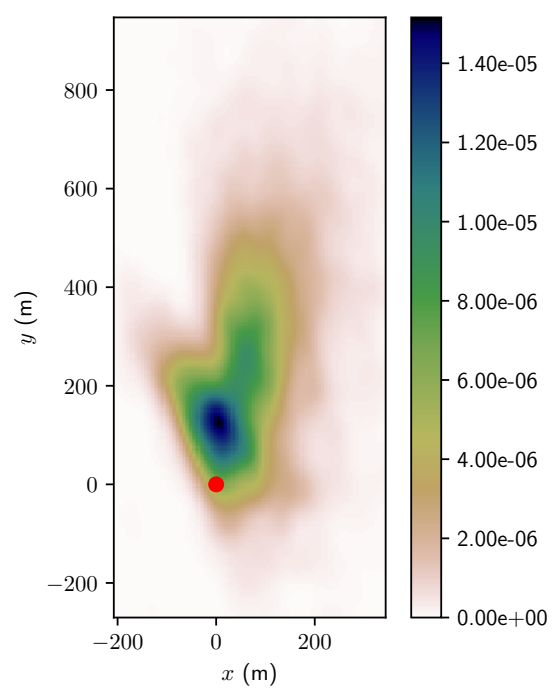

(b)

Fig. 4. Impact probability maps obtained by MC simulation for : (a) $h_{0}=$ $150 \mathrm{~m}$ and $V_{a_{0}}=30 \mathrm{~m} / \mathrm{s}$ with no wind; (b) $h_{0}=150 \mathrm{~m}$ and $V_{a_{0}}=20 \mathrm{~m} / \mathrm{s}$ with wind $\left(V_{w}=5 \mathrm{~m} / \mathrm{s}, \theta_{w}=3 \pi / 5 \mathrm{rad}\right)$

the two lateral modes by carrying out a helical descent. It is interesting to note that this phenomenon is emphasized by considering high UAV speeds as shown in Figure 4a where in this case, although the UAV starts its descent at maximum altitude, the lateral modes almost disappear. The occurrence of helical descents associated with these modes becomes very low due to the UAV's high aerodynamic speed, which therefore descends directly in straight glide path.

Finally, in the case with a wind blowing towards $y>0$ represented on Figure $4 b$, one can notice a very large deformation as well as a dispersion of the ground impact probabilities.

All these cases show the great large variations in the natures and shapes of the impact probability maps. In fact, they are multimodal, with very narrow maxima in some cases. Moreover, the probability of impact is almost close to zero at most of the points, which results in very sparse data. The size of the bounding area in which the impacts are contained can also vary greatly depending on the different cases.

For all these reasons, the analytical design of a model that could be used for fast computation of the probability of impact and of the resulting map for given values of the external parameters is not possible. This is why the design of surrogate models based on supervised learning has been considered and is proposed in the next section.

\section{SURROGATE MODEL DESIGN}

The parametric sampling of ground impact probability maps in a database developed in the previous section has a major advantage in terms of accuracy. This method therefore makes it possible to calculate the probability of ground impact beforehand, and subsequently the risk in the mission preparation phase when the aerodynamic conditions and external parameters are not completely defined.

However, these precise maps are only drawn up for a fixed sample set $\mathcal{A}_{s}$ of the external parameters previously defined during the development of the database. Increasing the sampling then amounts to generating more maps and requires the establishment of a much larger database. If this criterion is not discriminating for off-line calculation (because time and memory resources can be very large), it becomes a real issue if one wishes to assess the risk in real time (for reactive trajectory planning, for instance). Indeed, on-board memory and computational capabilities are limited and may not allow for online computation or update of such a database. It is thus difficult to envisage the use of the database for on-board risk calculation. Moreover, from a purely analytical point of view, it can be very useful to obtain a continuous function of the ground risk according to the external parameters.

To overcome these problems, the solution adopted consists in developing surrogate models through supervised learning. To do this, a training dataset must be created that contains sufficient information to allow the models to perform a precise regression of the probability maps. The function to be learnt therefore corresponds to a 6-parameter function composed by the 4 external parameters $\Theta=\left(h_{0}, V_{a_{0}}, V_{w}, \theta_{w}\right) \in \mathcal{A}$ as well as the coordinates in the impact plane $X=(x, y)$ :

$$
\operatorname{Pr}\{\text { impact }\}=g(\Theta, X)
$$

It is therefore possible to reuse the parametric database of ground impact points sampled over $\mathcal{A}_{s}$ that was generated in the previous section. This time, however, in addition to this, it is also necessary to choose a relevant sampling of the geometric coordinates $X$. The complexity of this problem lies in the fact that the probability of ground impact for a set of external parameters undergoes very strong variations as a function of the geometric coordinates. Furthermore, as explained previously, the probability of ground impact is almost zero everywhere except in the main modes, which makes this function difficult to learn by most of the surrogate models. 
The proposed idea is thus to develop a first model which will predict the bounds of the probability maps $\left(x_{\min }, x_{\max }, y_{\min }, y_{\max }\right)$ given a defined probability threshold, with respect to the external parameters $\Theta$.

A discrete subset $\mathcal{B}_{\Theta}$ of coordinates $(x, y)$ in the impact plane included within these bounds will then be defined in order to finely sample the estimated impact probability densities and to ensure that the training database contains sufficient information to capture variations of $g$.

A second model will be designed in order to learn the $g$ function within $\mathcal{B}_{\Theta}$ for any $\Theta$. By definition of the map bounds, the probability of impact on the ground outside this area will considered to be zero.

The development and implementation of the regression models associated with the map bounds and the assessment of the probability of impact are presented in the following section. The complete pipeline for generating impact probability maps from a set of external parameters is shown in Figure 5

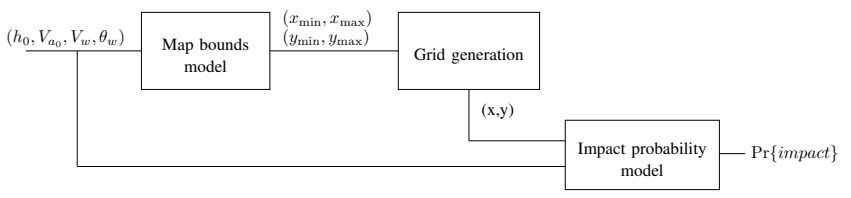

Fig. 5. Ground impact probability maps prediction pipeline

\section{A. Map bounds regression}

The magnitude of the probability of ground impact can vary greatly depending on external parameters. One of the most discriminating parameters is the initial altitude $h_{0}$. For very low altitudes, the UAV's impact trajectory will be direct a glide descent. Even if the parameters $\left(R_{0}, \gamma_{0}\right)$ are very scattered, the UAV will not even have time to perform an unstable helical descent before hitting the ground. As a result for these low altitudes, the impacts are very strongly localized in a reduced geometric area, which gives a very high probability of ground impact at this location.

At low altitude, wind direction also has a strong influence on the dispersion of the impact points. Indeed, even if the UAV should remain in a stable glide descent, a wind direction non collinear to the UAV velocity will tend to deviate the trajectories from their nominal straight glide path. For this reason, the angle $\theta_{w}$ also has a significant impact on the maximum impact probability value in a map.

Figure 6 highlights the influence of the two most sensitive parameters which are the initial altitude $h_{0}$ and the wind angle $\theta_{w}$. The $Z$ axis corresponds to the average of all the maximum values of each probability map respecting the axis values $\left(h_{0}, \theta_{w}\right)$ considering all $\left(V_{a_{0}}, V_{w}\right)$. We therefore notice a very strong increase in probability for altitudes below 40 meters with an initial wind angle of almost zero. In view of these abrupt variations, the bounds on the impact probability maps are determined by considering a rectangle containing all points with probability greater than a given threshold, the latter being defined at $2 \%$ of the maximum probability over a map. A padding of $10 \%$ of the size of the

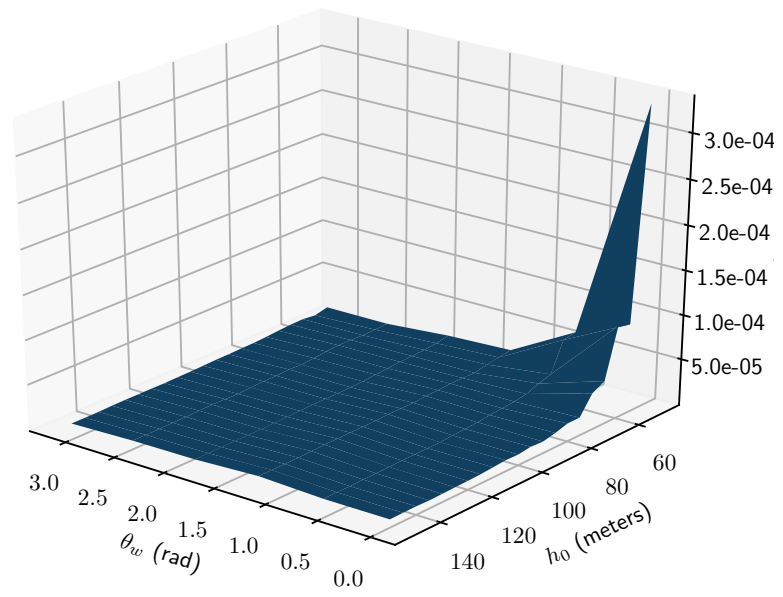

Fig. 6. Evolution of the average maximal value of the probability of impact in map as a function of $\theta_{w}$ and $h_{0}$

map was applied to ensure that there was no edge effect. A 1-layer perceptron network with 500 neurons in the hidden layer was chosen as a learning model which takes in inputs the 4 external parameters $\Theta=\left(h_{0}, V_{a_{0}}, V_{w}, \theta_{w}\right)$ and which returns in outputs the 4 map limits $\left(x_{\min }, x_{\max }, y_{\min }, y_{\max }\right)$ The database sampled on $\mathcal{A}_{s}$ developed in the previous section contains a regular tiling of the external parameters space. The regression model of the map bounds was therefore trained on these $\operatorname{card}\left(\mathcal{A}_{s}\right)=2,250$ parameters. To validate the data, a second database was generated using a Latin Hypercube Sampling (LHS) of the external parameters space, $\mathcal{A}_{t} \subset \mathcal{A}$. This sampling has the advantage, contrary to random sampling, of guaranteeing a good representation of the variability of the data.

Figure 7 shows the learning curve of the network. The final performance of the validated model can be quantified by looking at the relative average error in terms of range which is $9.7 \%$ along the $x$ axis and $4.8 \%$ along the $y$ axis.

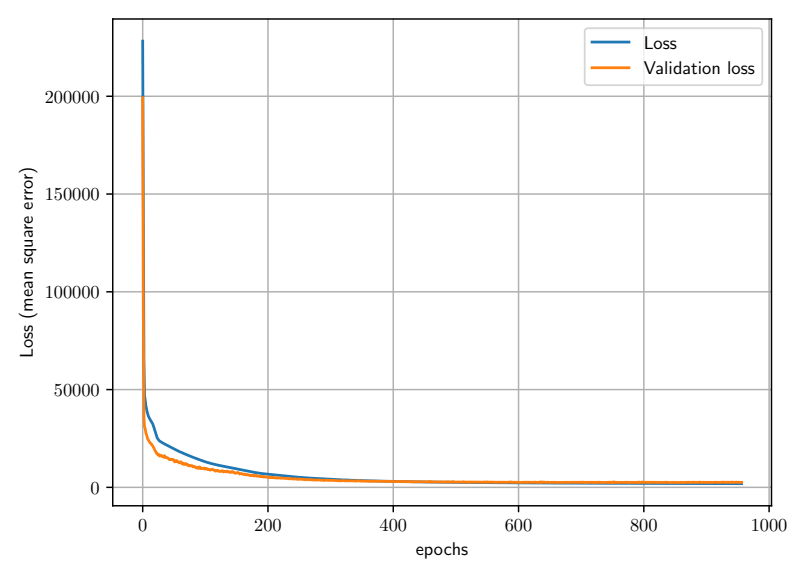

Fig. 7. Training curves for regression of bounds on probabilistic maps of impact

Although defined by a relative probability, these bounds 


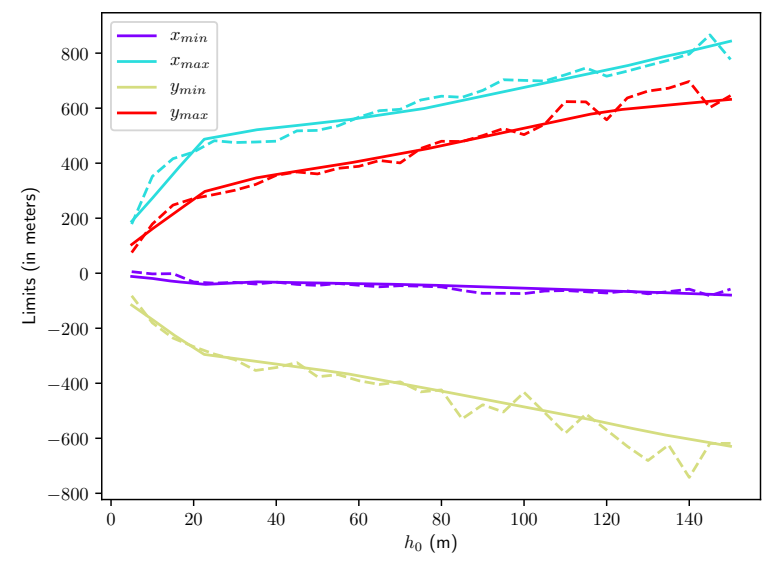

(a)

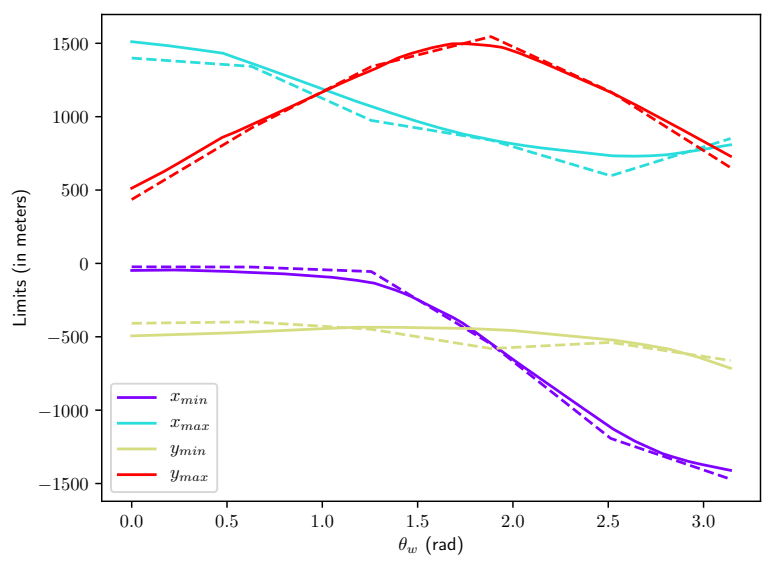

(b)

Fig. 8. Evolution of the bounds of the impact probability maps as a function of a variable of interest: (a) variation w.r.t. $h_{0}$ with $\left(V_{w}, \theta_{w}, V_{a_{0}}\right)=$ $(0,0,25)$; (b) variation w.r.t. $\theta_{w}$ with $\left(V_{w}, V_{a_{0}}, h_{0}\right)=(10,25,150)$

can be interpreted as limits of the UAV impact area. These results can therefore be used as they stand to define certain operational templates.

Figure 8a shows the influence of bounds with initial altitude $h_{0}$. The model prediction is represented by the continuous lines while the actual values are represented by the dashed lines. It can be seen that in the windless case, the evolution of the crash distribution is almost linear. For low altitudes, the scattering of the impacts decreases drastically and makes the prediction sometimes incorrect. However, considering the missions for fixed-wing UAVs, altitudes below 50 meters are rarely considered.

Figure $8 b$ shows the influence of wind direction on the distribution of impacts and shows the very strong deformations induced on the impact figure, especially on limits $x_{\min }$ and $y_{\max }$.

\section{B. Probability regression}

The problem of regression of the probability of ground impact contains many difficulties that need to be properly managed before developing any surrogate model. Several results from the analysis of the geometric bounds of the probability maps can be reused in the ground impact probability regression analysis. By considering the impact probability of function (5), several characteristics can be mentioned:

- The $g$ function is very sparse, this is related to the fact that the impact probabilities are very concentrated with respect to the $(x, y)$ variables.

- The function $g$ is strongly non-linear in $\theta_{w}$ and $h_{0}$, leading to very high probability peaks for low values of $h_{0}$. This produces very unbalanced data which makes the optimization problem complicated.

The advantage of using a kernel estimator is that it allows resampling the probability density estimate without changing the precision of the estimate. This characteristic thus makes it possible to have as much data as necessary for each map with respect to the variables $(x, y)$.

To constitute the training set intended to learn the function $g$, it is necessary to well define the design of experiment associated to the parameters $(\Theta, X)$. Since the sampling of external parameters $\Theta$ was computed in the database over $\mathcal{A}_{s}$, this set should be expanded using a relevant sampling of the geometric parameters $X$.

For each impact probability map associated with the external parameters $\Theta$, the $X$ sampling is defined by a discrete subset $\mathcal{B}_{\Theta}$ of the impact plane, that corresponds only to the area that actually contains information about $g$. The previously calculated map bounds are therefore very useful in order define the extrema of this subset. The second parameter necessary for its definition is the step along $(x, y)$ axis, $\mathcal{B}_{\Theta}$ being chosen as a regular grid. Since variations in $g$ are highly dependent on $\Theta$, each map is sampled on 30 points along the $x$-axis (the number of sample points along the $y$-axis resulting from proportionality). By flattening the sampled maps and concatenating them into a $1 \mathrm{D}$ vector, we finally obtain a training set of more than 2 millions of points. Following the same reasoning, the validation set for the impact probability density regression is generated by expanding the LHS of external parameters $\mathcal{A}_{t}$.

Given the complexity of the problem as well as the large amount of training data available, a multi-layer perceptron network is used.

The data pre-processing phase is fundamental for the training of the network. Indeed, the cost function used for the optimization of the network being the mean square error (MSE), the raw use of the normalized data would lead to a very strong weighting of the high probability points, totally vanishing the less strong probability variations. Given the sensitivity analysis performed on the variables $\left(h_{0}, \theta_{w}\right)$ and presented in Figure 6, low altitude maps with collinear wind and UAV velocities would be much more weighted than high-altitude maps. The transformation applied to the data 


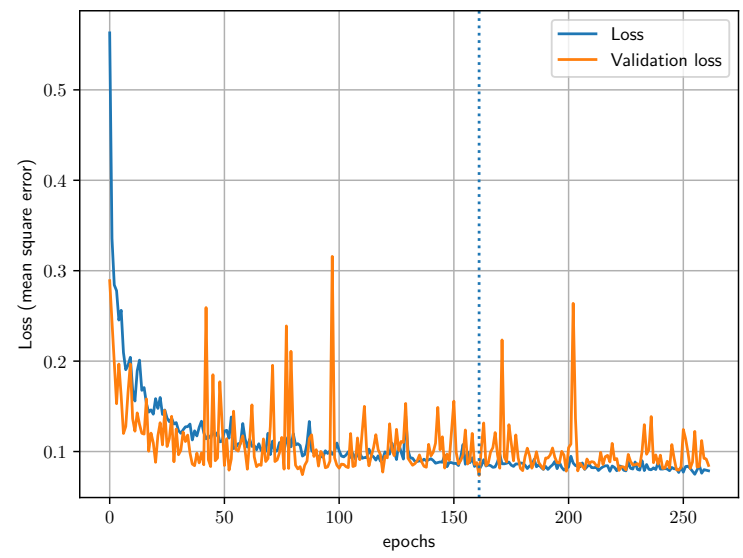

Fig. 9. Learning curves for probability of impact function regression

is therefore

$$
g_{\text {norm }}(\Theta, X)=h_{0} \cdot\left(\theta_{w}+2\right) \cdot g(\Theta, X)
$$

Finally, given the small number of input features in the network, it may be advisable to artificially increase the number of entries by considering the sensitivity analyses carried out to guide the training phase of the network. Two kernels were introduced to catch the output probability dependencies based on a sensitivity study: $x_{1}=\exp \left(h_{0} / 6\right)$, $x_{2}=\theta_{w} \cdot h_{0}$.

The structure finally chosen for the model contains 3 hidden layers composed of 100, 500 and 100 neurons respectively. The learning curve of the network is shown in Figure 9 One can use the objective metric defined by (7) to evaluate the relevance of the prediction $\hat{g}$ of $g$.

$$
\epsilon_{t}=\frac{1}{\operatorname{card} \mathcal{A}_{t}} \sum_{\Theta \in \mathcal{A}_{t}} \sum_{X \in \mathcal{B}_{\Theta}} \frac{1}{\operatorname{card} \mathcal{B}_{\Theta}} \frac{|g(\Theta, X)-\hat{g}(\Theta, X)|}{\max _{X \in \mathcal{B}_{\Theta}} g(\Theta, X)}
$$

Finally, the error obtained on the validation set amounts to $\epsilon_{t}=2.25 \%$. This metric, although somewhat optimistic, provides an objective quantification of the model's results. It is noted that this error would be $100 \%$ if the estimated probability obtained is in perfect opposition to the real value with respect to the max value of the map.

Two examples of predictions on cases presented before are given in Figures 10 and 11. These visual results support the objective metric of the relevance of the predictions. The contribution of the corresponding error for each one of the maps is respectively $\epsilon=2.10 \%$ and $\epsilon=2.82 \%$ The relative error corresponding to each bin on the map is shown in Figures 12 and 13. We can see that despite some spikes of error of around $30 \%$, the main modes are still correctly estimated and the error in these modes is less than $15 \%$ on average.

The complete pipeline taking into account data preprocessing, network predictions and the adaptation of the resulting probability map produces a result in a few milliseconds.

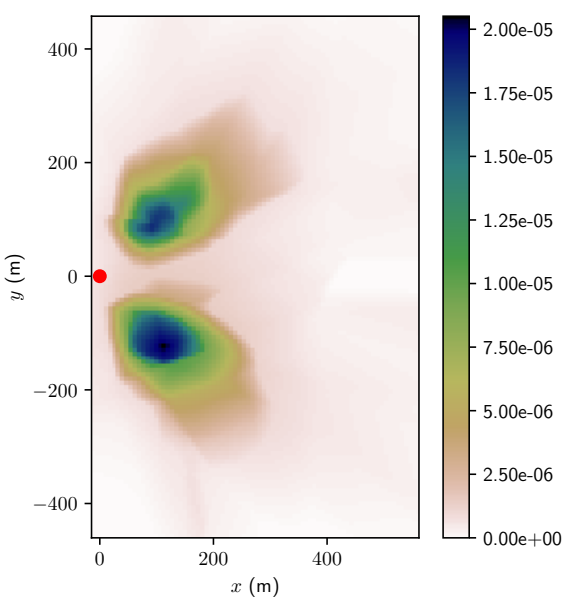

Fig. 10. Impact probability map predicted by the model with no wind, $h_{0}=150 \mathrm{~m}$ and $V_{a_{0}}=20 \mathrm{~m} / \mathrm{s}$

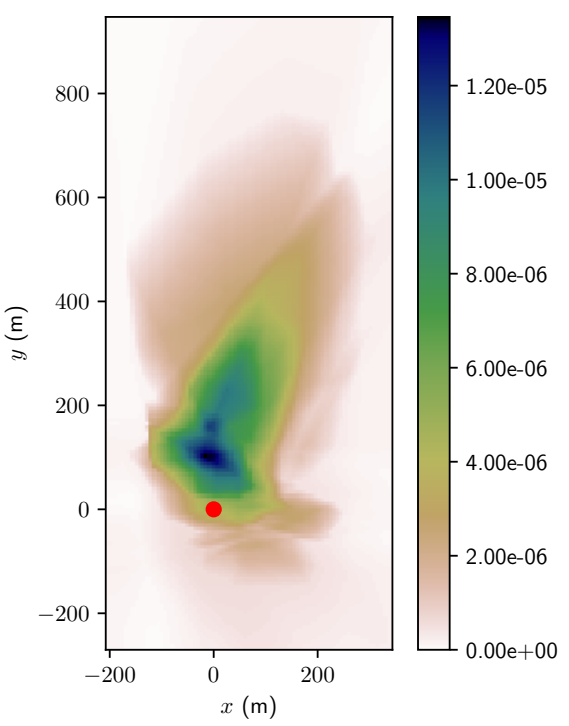

Fig. 11. Impact probability map predicted by the model with $h_{0}=150 \mathrm{~m}$, $V_{a_{0}}=20 \mathrm{~m} / \mathrm{s}$ with wind $\left(V_{w}=5 \mathrm{~m} / \mathrm{s}, \theta_{w}=3 \pi / 5 \mathrm{rad}\right)$

\section{CONCLUSIONS}

The calculation of the ground impact probability in case of failure for a fixed-wing UAV represents one of the fundamental points in quantitative ground risk determination. This paper first presented a precise method for generating a database of impact probability maps by $\mathrm{MC}$ based on a relevant sampling of the parameter space. This method can allow for an off-line risk evaluation for preliminary analysis of UAV missions (e.g. mission preparation). In a second step, a method based on supervised learning by neural network trained on the previous database has allowed to generate these ground impact probability maps in a continuous way with respect to the parameters of 


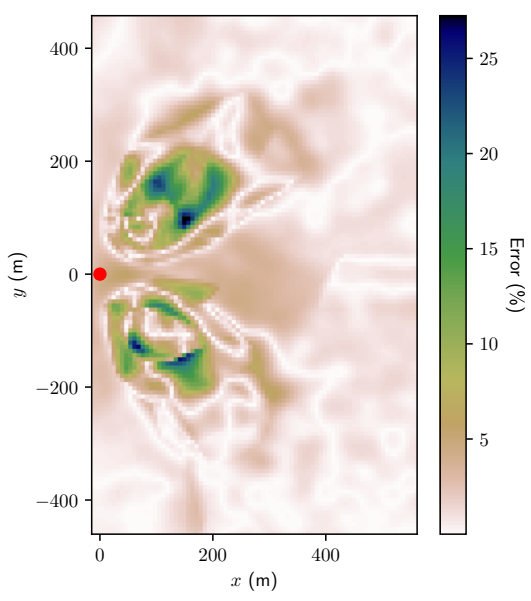

Fig. 12. Relative error map between model predictions and MC simulation with no wind, $h_{0}=150 \mathrm{~m}$ and $V_{a_{0}}=20 \mathrm{~m} / \mathrm{s}$

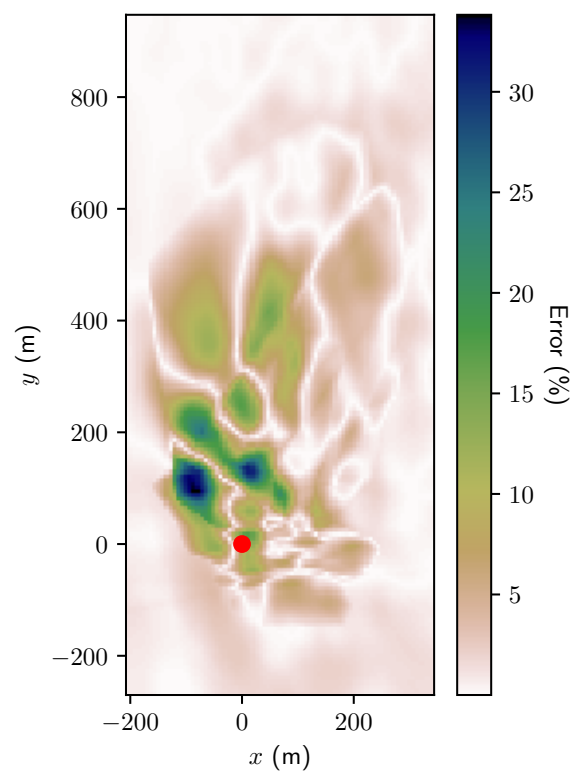

Fig. 13. Relative error map between model predictions and MC simulation with $h_{0}=150 \mathrm{~m}, V_{a_{0}}=20 \mathrm{~m} / \mathrm{s}$ with wind $\left(V_{w}=5 \mathrm{~m} / \mathrm{s}, \theta_{w}=3 \pi / 5\right.$ $\mathrm{rad})$

interest, including wind. The calculation time and memory requirements being drastically reduced, this method can allow for an online risk assessment.

Future work will involve this tool in the calculation of risk in Long Range Operations (LRO). An important step will also be to quantify the limit errors in comparison with MC simulations in order to guarantee the reliability of the model.

Real-time reactive navigation methods that take into account the ground risk level will also be explored.

\section{ACKNOWLEDGMENT}

This work has been supported by French DGAC in the context of the research partnership PHYDIAS with ONERA for safety improvement of UAVs.

\section{REFERENCES}

[1] S. Bertrand, N. Raballand, F. Viguier and F. Muller, "Ground Risk Assessment of Long-Range Inspection Missions of Railways by UAVs", International Conference on Unmanned Aicraft Systems, 2017.

[2] R. Clothier, R. Walker, N. Fulton and D. Campbell, "A Casualty Risk Analysis for Unmanned Aerial System (UAS) Operations over Inhabited Areas," 12th Australian International Aerospace Congress, 2007.

[3] A. La Cour-Harbo, "Quantifying risk of ground impact fatalities of power line inspection BVLOS flight with small unmanned aircraft", International Conference on Unmanned Aircraft Systems, 2017.

[4] S. Bertrand, N. Raballand, F. Viguier, "Evaluating Ground Risk for Road Networks Induced by UAV Operations", International Conference on Unmanned Aicraft Systems, 2018.

[5] A. Washington, R.A. Clothier, J. Silva, "A review of unmanned aircraft system ground risk models", Progress in Aerospace Sciences, 2017.

[6] A. La Cour-Harbo, "Ground impact probability distribution for small unmanned aircraft in ballistic descent", Reliability Engineering and System Safety, 2017.

[7] P. Wu, R. Clothier, "The development of ground impact models for the analysis of the risks associated with Unmanned Aircraft Operations over inhabited areas ", International Probabilistic Safety Assessment and Management Conference and the 2012 Annual European Safety and Reliability Conference, 2012.

[8] A. Poissant, L. Castana, H. Xu, "Ground Impact and Hazard Mitigation for Safer UAV Flight Response", International Conference on Unmanned Aircraft Systems, 2018.

[9] B. Levasseur, S. Bertrand, N. Raballand, F. Viguier, and G. Goussu, "Accurate Ground Impact Footprints and Probabilistic Maps for Risk Analysis of UAV Missions", IEEE Aerospace Conference, 2019.

[10] A. De Marco, E.L. Duke and J.S. Berndt, "A General Solution to the Aircraft Trim Problem", AIAA Modelling and Simulation Technologies Conference and Exhibit, 2007.

[11] M.P. Wand, M.C. Jones, "Kernel smoothing", Monographs on Statistics And Applied Probability, 1995.

[12] M.P. Wand, M.C. Jones, "Multivariate plug-in bandwidth selection", Computational Statistics, 1994

[13] T. Duong, M. Hazelton, "Plug-in bandwidth matrices for bivariate kernel density estimation", Journal of Nonparametric Statistics, 2003

[14] T. Duong, "ks: Kernel Density Estimation and Kernel Discriminant Analysis for Multivariate Data in R", Journal of Statistical Software, 2007. 